\title{
Sur le grand huit en compagnie du Conseil fédéral
}

\section{Yvonne Gilli}

Dre méd., présidente de la FMH

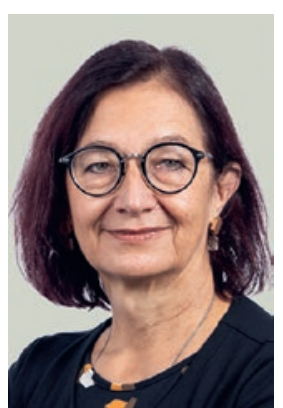

Les vagues de la pandémie rappellent un tour en montagnes russes, avec plusieurs particularités toutefois: on monte plus vite qu'on ne descend, et les risques sont réels. Régulièrement, certains sont éjectés et blessés, voire tués. Et plus on monte haut, plus il y a de morts. Il y a trop peu de harnais de sécurité; on les a commandés, mais il se pourrait qu'on attende longtemps avant d'en recevoir suffisamment. Après plus d'une année, nous ne savons toujours pas quels sont les virages les plus dangereux ni quelles sont les précautions les plus efficaces. Tous s'agrippent à leur siège tandis que le train de wagons est freiné une fois plus, une fois moins, et chemine à une allure d'escargot - les passagers s'impatientent. Nous sommes en avril 2021 et l'exploitant du parc répond aux critiques toujours plus vives des passagers frustrés, coincés dans leurs wagons. Malgré l'augmentation du nombre de cas, il serait temps de desserrer les freins et d'assouplir les mesures. Ce faisant, notre exploitant brûle quatre des cinq stops qu'il a lui-même installés. Agit-il par crainte que les gens perdent patience? Non, rétorque-t-il, la plupart se comportent de manière responsable, et cela pourrait fonctionner, «si chacun y met du sien».

\section{Ce qui pourrait passer pour un compliment} à la population ne fait que reporter sur elle la responsabilité des nouvelles contaminations.

Ce qui pourrait passer pour un compliment à la population ne revient qu'à reporter sur elle la responsabilité des nouvelles contaminations, et à ignorer les obligations de l'exploitant. Admettons: ce qui compte n'est pas tant ce que les autorités décident que ce qu'elles-mêmes et la population en font concrètement. En médecine, on parlerait d'adhésion au traitement, une thérapie n'étant jamais aussi bonne que la manière dont le patient l'applique. De la même façon, les décisions politiques ne sont efficaces que dans la mesure où la population les accepte. Pour autant, cela ne diminue en rien l'importance d'un bon plan thérapeutique, bien au contraire. En médecine comme en politique, tout l'art consiste à allier qualité de la stratégie et qualité de la mise en œuvre.

Que faire, dès lors, quand l'adhésion au traitement faiblit? On peut s'en remettre à l'autorité brute, comme en
1710 en Prusse orientale, où tout manquement aux mesures contre la peste se soldait par une pendaison [1]. Mais on peut aussi faire preuve d'autocritique et passer en revue chaque étape pour comprendre où l'on n'a pas su convaincre. A-t-on choisi les bonnes mesures, et les a-t-on introduites de manière adéquate? Quels ont été les effets, et les effets secondaires de ces mesures? Quels coûts engendrent-elles, pour quel degré d'efficacité? Et que nous apprend le déroulement du traitement

\section{Le mécontentement peut signifier que le plan} de traitement est difficilement applicable.

jusqu'ici; avons-nous épuisé toutes les possibilités? Le mécontentement de la population montre-t-il que nous avons failli à notre devoir de proposer la thérapie dotée du meilleur rapport bénéfice-risque? Que notre plan se traduit mal dans la pratique?

L'exploitant du parc a choisi une troisième voie: selon le volume des protestations, il freine une fois davantage, une fois moins. Lui-même solidement attaché, il exhorte les gens à bien se tenir. Il agit à la manière d'un médecin qui administrerait toujours le même médicament en variant constamment le dosage, et qui sermonnerait le patient pour qu'il le prenne correctement. Lorsque la dose diminue, les effets secondaires diminuent rapidement, mais l'efficacité aussi, et inversement. Après un an de pandémie, nous devrions savoir quel type de virage présente quel type de risques, et être à même d'agir de manière plus ciblée. Nous devrions avoir une stratégie de test qui mérite ce nom. Et il faudrait aider la population à redescendre sur la terre ferme en allouant les moyens nécessaires pour une vaccination à large échelle au cabinet. Il faudrait disposer de certificats de vaccination adéquats ainsi que d'un plan détaillant ce qu'il conviendra de faire si d'aventure il nous fallait à nouveau davantage de harnais de sécurité. Tout ceci relèverait en fait de la responsabilité de l'exploitant du parc. Alterner les coups de frein et d'accélérateur, en espérant que tous s'agrippent du mieux qu'ils peuvent jusqu'à la livraison d'un nombre suffisant de harnais de sécurité, n’est pas une stratégie adéquate.

\section{Référence}

1 Fox W. Compliance of patients and physiscians: experience and lessons from tubercolosis. Br Med J. 1983;287(6384):33-5. 